\title{
Meanings of the equals sign in upper-level undergraduate problem solving
}

\author{
Dina Zohrabi Alaee, ${ }^{1}$ Eleanor C. Sayre, ${ }^{1, *}$ and Scott V. Franklin ${ }^{2}$ \\ ${ }^{1}$ Department of Physics, Kansas State University, Manhattan, Kansas 66506, USA \\ ${ }^{2}$ Department of Physics, Rochester Institute of Technology, Rochester, NY
}

\begin{abstract}
The conceptual meaning behind the equals sign depends on its use and can show the cultural significance of different practices in solving physics problems. We use Sherin's symbolic forms to investigate the conceptual and cultural meanings of the equals sign across physics contexts. In this study we build and validate a rubric to classify physics students' use of the equals sign in written homework as assignment, causality, definitional, balancing, calculate, or hybrid. We then analyze students' solutions in their written homework in an upper-division electrostatics course and compare them to textbook solutions.
\end{abstract}

\section{INTRODUCTION}

It is popular, at times, to claim that mathematics is the language of physics. Much literature around problem solving in physics focuses on how students use mathematics to instantiate physics concepts. Taking up the metaphor of math as language, we examine "grammar" on a minute level to investigate the particular dialect of mathematics spoken in physics classes.

Writing in the Knowledge-in-Pieces tradition, Sherin's symbolic forms framework [1, 2] links mathematical equations to intuitive conceptual ideas. This framework explains how students create, describe and evaluate equations to understand physics concepts and provides a small-grain size for analysis into the grammar of students' mathematics use in physics. We take up the idea of symbolic forms and focus on just one aspect: the conceptual meanings behind the central symbol in every equation, the equals sign. Specifically, we investigate the different conceptual meanings of the equals sign within each equation sentence that emerges in physics problem solving and how these meanings shift in relative frequency across contexts and speakers. In our prior work[3], we developed a classification scheme for these mathematical sentences and equals sign use and applied it to textbooks drawn from across the four-year undergraduate curriculum. In this paper, we examine how upper-division undergraduate students use these mathematical sentences in their electromagnetic fields course, and compare the students' "dialect" to the one presented in the solution manual for the course textbook.

\section{CATEGORIES}

In [3] we examined physics textbooks from across the undergraduate curriculum to identify categories of equals sign use. A team of researchers including undergraduates, graduate students and physics faculty came to consensus on five categories of equals sign use and showed

* esayre@ksu.edu how the frequency of different categories varies by physics topic and course level.

A more thorough description of these categories and their development is available in [3]; here we briefly restate the categories: assignment, causality, definitional, balancing, and calculate.

Causal: The causal equals sign indicates a quantity, usually represented as a single variable caused by term $(\mathrm{s})$ on the right-hand side. For example, $\oint \vec{E} \cdot d \vec{A}=\frac{Q_{e} n c}{\epsilon_{0}}$ indicates the creation of an electric field $\vec{E}$ by existing charges.

Definitional: A definitional equals sign, sometimes indicated with $\equiv$, establishes a fundamentally new quantity. For instance, the inertial mass $m$ is defined - conceptually and operationally — as $m \equiv|\vec{F}| /|\vec{a}|$.

Assignment: In this category, a variable is assigned to an expression for some particular use. For example, situations in which a variable is equal to collection of constants for simplification, e.g. $y_{0}+v_{0 y} t+\frac{1}{2} a_{y} t^{2}=0+(15 \mathrm{~m} / \mathrm{s}) t+\frac{1}{2}\left(-9.8 \mathrm{~m} / \mathrm{s}^{2}\right) t^{2}$, are coded as assignment. The assignment category also includes assigning a new variable to a combination of existing variables, as in the case of a damped harmonic oscillator: $\gamma=\frac{c}{2 m}$ and $\omega_{0}^{2}=\frac{k}{m}$.

Balancing: Balancing describes a dynamic equilibrium between variables. For example, the 1-D wave $\frac{\partial^{2} \psi(x, t)}{\partial t^{2}}=c^{2} \frac{\partial^{2} \psi(x, t)}{\partial x^{2}}$ shows a dynamic balance between spatial and temporal derivatives. Unlike other categories, the order in which this equation is written does not affect the meaning.

Calculation-Math: The calculation equals sign is used in a manner similar to a calculator's " $=$ " button and appears primarily during problem solving.

In this study we apply our classification scheme to compare textbooks' problem solutions with that of students'. We do not observe any causality type of equals sign across the data, a result perhaps of the problem-solving process. This could have been an artifact of the problems we chose to analyze; however, its low frequency in textbooks suggests a similar low frequency in students' problem solving. We note the appearance in students' solutions of a 
sixth category. Students used assignment and calculate simultaneously, usually when seeking a shortcut in the problem solving process, a use we term hybrid:

Hybrid: Hybrid is a representation of assignment and calculate occurring simultaneously. This is generally seen when students find a shortcut in the problem solving process. For example, to find the net force the southern hemisphere in a uniformly charged sphere exerts on the northern hemisphere in terms of the radius, students write down the force in $z$ direction

$$
\begin{gathered}
F_{z}=\frac{3}{\epsilon_{0}}\left(\frac{Q}{4 \pi R^{3}}\right)^{2} \int_{0}^{R} r^{3} d r \int_{0}^{\pi / 2} \cos \theta \sin \theta d \theta \int_{0}^{2 \pi} d \phi \\
\stackrel{\text { Hybrid }}{=} \frac{3}{\epsilon_{0}}\left(\frac{Q}{4 \pi R^{3}}\right)^{2}\left(\frac{R^{4}}{4}\right)\left(\left.\frac{\sin ^{2} \theta}{2}\right|_{0} ^{\pi / 2}\right)(2 \pi) .
\end{gathered}
$$

Students assign a variable and solve the problem by calculating some parts in their mind and combine them for the solution.

We emphasize two limitations in our study. First, the focus on the equals sign intentionally ignores other symbols as indicators of dialect. Second, we emphasize the importance of context in our interpretations, and classification of a particular symbol depends sensitively on its relation with the surrounding text and other equations.

\section{DATA SELECTION}

Two sets of data are drawn from an upper-division course in an Electromagnetic Fields (E\& M) at Kansas State University. This course is available each fall and typically covers the first half of Griffiths' textbook[4] (the most popular book in the US market for this course). The 50-minute class meets four days a week and is composed roughly of equal parts tutorial, group problem solving, and interactive lecture.

Our first data set is composed of students' written homework solutions. Homework in this class contains problems drawn from the Griffiths' textbook as well as other sources, such as instructor-generated problems. The second data set is composed of the "official" solutions to a subset of those problems from the Griffiths' textbook solutions manual, augmented by additional problems in the solutions manual that were not assigned in class. For both data sets we first applied and amended our classification scheme. Once a stable set of categories was in place we invited additional researchers to separately code students' solutions. These researchers applied the categorization scheme to a randomly selected set of $10 \%$ of the entire data set; inter-rater reliability testing resulted in a $63 \%$ agreement. After subsequent clarification and refinement, we repeated this procedure with an additional $20 \%$ of the data set and reached $100 \%$ agreement.

\section{HOMEWORK DATA}

The context of this study is an Electromagnetic Fields (E\& M) course at Kansas State University. To begin the study 30 problems drawn from 11 problem sets throughout the semester were coded. The data were coded consisted of instructor-generated problems and Griffiths' textbook problems. We selected problems to have broad range of topics in the course with non-trivial solutions likely to contain multiple types of equals sign. Three student solutions per problem were chosen; criteria for selection included legibility, correctness of solution and sufficient difference from the solutions manual to preclude blind copying.

Students used many representations (words, graph, equations, etc.) and different problem-solving strategies to solve similar problems. We find that students are most likely to use just three types of equals sign while solving E\&M homework problems: definitional $(D)$, assignment $(A)$, and calculation $(M)$.

Students rarely use balancing, causality and hybrid equals signs while solving E\&M problems. We suspect that this is a consequence of both the course level and problem solving process. Our textbook analysis [3] suggested that causality equals sign are more prevalent at the introductory level and students may mirror dialect from the textbook.

Students vary in their problem solving strategies. Some skip mathematical steps while others include each step in great detail. The latter mostly used one kind of the equals sign several times in sequence. The statistical order of appearance of equals sign can be represented graphically; light and dark gray arrows in Fig.1 show the directionality of movement, with the arrow thickness indicating the relative frequency of each use. The curved black arrows indicate successive uses of the same sign; the thick arrows in assignment and calculate show their predominance.

Students more often move from definition to assignment to calculate, a clockwise motion (light gray arrows) as represented in Fig. 1. Students begin solving a problem by translating the problem statement into a symbolic form, then recall equations, apply the conditions of the problem and, finally, solve the problem.

\section{COMPARISON TO SOLUTION MANUALS}

We noticed that students' solution of instructorgenerated problems is qualitatively different compare to students' solution of Griffiths-generated problems. Students have plenty of previous knowledge and ideas that they bring together when solving physics problems. We investigated further to see if the students' solution of Griffiths-generated problems are similar to Griffiths' solution of Griffiths-generated problems. To achieve this, we started study two, which is comparing the students' solution of Griffiths-generated problems with Griffiths' 


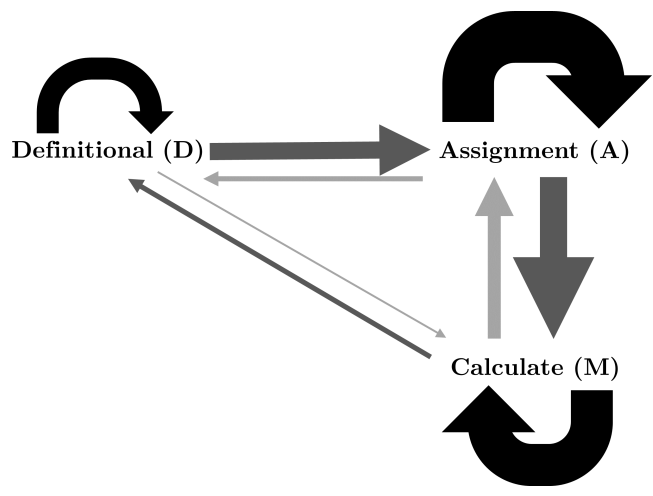

FIG. 1. Students' movement between different categories.

solution of Griffiths-generated problems. We can then compare the performance and see how closely students' solution aligned with the Griffiths' approach.

The second data set is derived from the textbook solutions manual to homework problems. There is a total of 12 homework problems drawn from the Griffiths textbook, with each problem consisting of two to four sections. In comparison to students' solutions the solutions manual's solutions are much shorter, with fewer steps compressed into a smaller physical space and less verbal narration or use of multiple representations. In some problems Griffiths explains the problem statement in text and recalls equations from previous problems. The majority of solutions are more abstract than those of students. Like the students, Griffiths uses definitional $(D)$, assignment $(A)$, and calculation $(M)$ equals sign more than other categories.

Figure 2 shows the frequency of each path between students' solutions and Griffiths' solutions manual. There is asymmetry between MD (calculate to definitional) and DM (definitional to calculate), MA (calculate to assignment) and AM (assignment to calculate), etc. Across our corpora, we notice the definitional type of equals sign usually appears at the beginning of the solution, followed by assignment and calculate types. We can see students are consistent in their approaches to solving problems with similar structure. This asymmetry is more apparent in Figure1, where we have represented directionality. These results demonstrate that students and Griffiths both prefer to make transitions in a clockwise rather than counterclockwise sense in this diagram: DA is bigger than $\mathrm{AD}$, etc.

We use network analysis [5] to compare usage patterns of both students' and Griffiths' solutions to represent the relationships between categories and the steps of problem solving. In our networks, shown in Figure 3, nodes indicate different categories of equals sign while edges indicate sequential usage which we interpret as interactions between types. Figure 3 includes self-connections for each node indicating sequential uses of the same sign.

We use the idea of centrality to gain insight into the relative importance of the nodes (equals sign types). Cen-

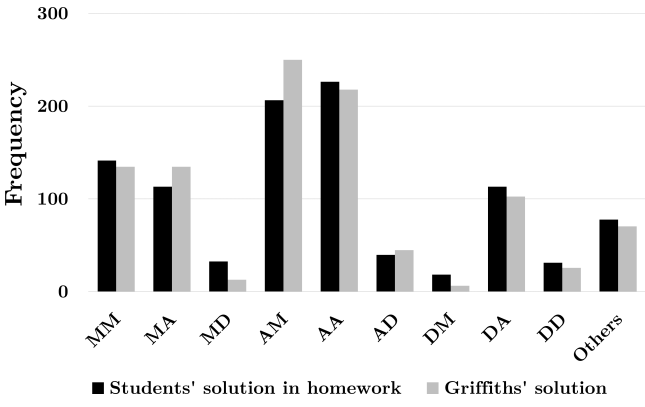

FIG. 2. Frequency of each path between students' solutions and Griffiths' solutions manual.

trality analysis characterizes the structure of the relationships between nodes within the network to see which nodes are most "important" or central to a network. Here we consider three measures of centrality: degree, closeness and betweenness. Degree centrality identifies nodes with the largest number of ties to other nodes; betweenness is a measure based on shortest paths and closeness measures the mean distance from a node to other edges.

Several features are represented in these networks. Figure 3 indicates that even though students took many different approaches to problem solving, the connections between definitional, calculate, hybrid, and assignment are more important than other categories. Furthermore, Griffiths tends to omit some mathematical steps, resulting in a less connected graph, yet the relative connections are similar. We note that students utilized the balancing type while Griffiths did not. Students' process of generating knowledge, their writing style, and the way that they sorted ideas in E\& M provided them some balancing type of equals sign which we rarely saw in Griffiths' solutions. Griffiths' solutions have a clear mathematical explanation, which do not include the balancing type that can help students to understand the physical concepts.

Figure 3 shows that the assignment node has many links in both networks, thus a higher centrality degree for both in-degree \& out-degree. The closeness centrality usually implies that all paths should lead from a node to everywhere else. It is clear that balancing in Griffiths' network and balancing and hybrid in the students' network are far-removed from the rest of the network. With this more complete measure of betweenness centrality, the assignment type of equals sign is the most important node in both networks, with the calculation equals sign less important. Since each problem has several parts, this can be the evidence that both students and Griffiths may have to go back and check their prior solution. Then, in the next part, they can assign the value in to the next part and calculate final answer. Overall, the students' solution component is largely the same with Griffiths' solution across Griffiths-generated problems. The students largely performing the way that a normative expert suggested they should perform. 

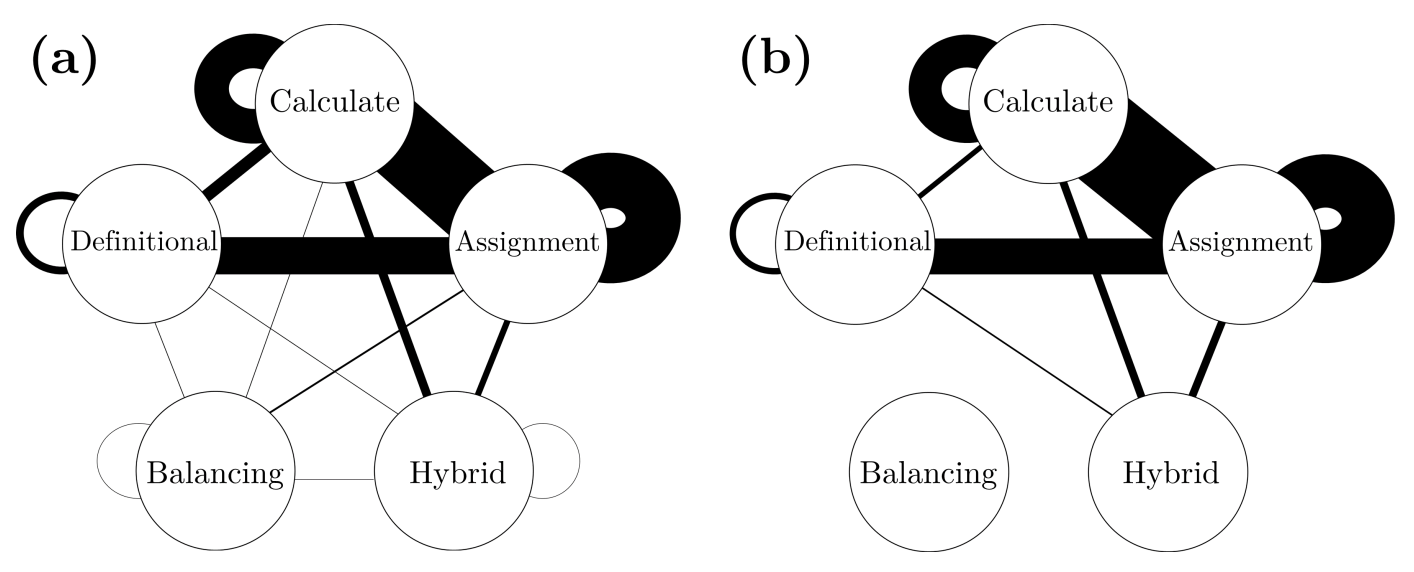

FIG. 3. (a) Students' solution network. Categories are represented by a circle and connecting lines represent the order of steps students use to solve problems. (b) Griffiths' solution manual's network.

\section{DISCUSSION}

This work explores students' use of equals sign while solving problems in an undergraduate level physics E\& M course. We have identified six categories of equals sign, discussed how these types of equals sign convey different conceptual physical meanings and identified frequency and nature of use in students' and textbook problem solutions. Comparing students' solution to Griffiths' solution shows allows us to check if their solution paths are similar to a normative path, while analysis of their solutions to problems outside of Griffiths helps us describe their solutions absent (ubiquitous) solution manual solutions. Network analysis indicates that the most important type (node), common in both students' and Griffiths' solution, is the assignment type. While the particular procedural steps differ across students and problems, this result is robust.

These results highlight the importance of understanding how undergraduate students think about equality and use the equals sign. As the use of equals sign is one indicator of the dialect of math used in physics, we can explore differences in how students, textbooks, and instructors use this indicator to better study the grammar of math in physics.

Focusing attention on the structure of the equations involving the equals sign leads to an understanding of an equation's underlying meaning which can help illumi- nate the dialect of mathematics used in physics. This could help drive students' conceptual understanding instead of equation memorization. Finally, thinking more deeply about the equals sign may help instructors consider alternative presentations of equations. Drawing direct applications to instruction from this study would be premature, however; a valuable next step in this research would be a study connecting fundamental and applied research (e.g. curriculum development) with an eye on developing instructional goals.

This analysis is a part of a larger work in which equals signs are studied in a variety of undergraduate level physics textbooks[3]. In the future, we will consider how physicists use the equals sign in their work as well. For instance, we will investigate the specific role a physics instructor goes through in providing assistance to students in order that we can understand the conceptual meaning of equals sign during physics problems solving. In addition to examining the formal written dialect of math in physics, we plan to expand our fundamental work to more informal spoken dialects as well.

\section{ACKNOWLEDGMENTS}

The authors acknowledge the support from the KSU Department of Physics. They are also grateful to Bahar Modir and Natasha Graham for assistance with the interrater reliability. This work was funded in part by the NSF under grant number DUE-1430967.
[1] B. L. Sherin, Cognition and Instruction 19, 479 (2001).

[2] B. Sherin, Journal of Research in Science Teaching 43, 535 (2006).

[3] E. C. S. Dina Zohrabi Alaee, Kellianne Kornick and
S. Franklin, Submitted (2017).

[4] D. J. Griffiths, Prentice Hall 93, 95 (1999).

[5] Eric D. Kolaczyk âĂć Gábor Csárdi, Statistical Analysis of Network Data with $R$ (Springer New York). 